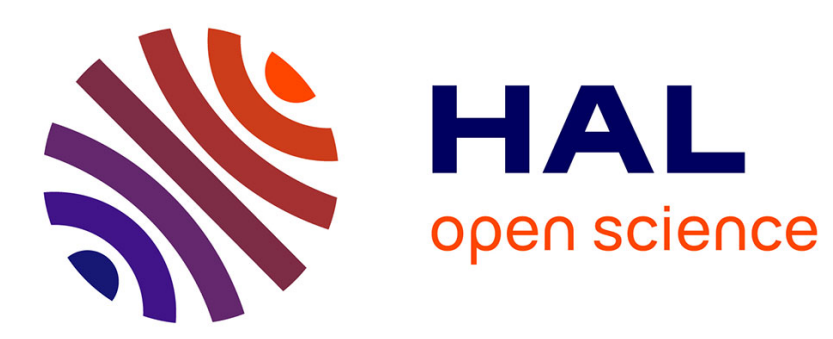

\title{
Phosphoroscope spectrophotographique destiné à la mesure des durées de vie des luminescences retardées de faible intensité
}

\author{
Yvan Rousset, Francois Dupuy, Robert Lochet
}

\section{> To cite this version:}

Yvan Rousset, Francois Dupuy, Robert Lochet. Phosphoroscope spectrophotographique destiné à la mesure des durées de vie des luminescences retardées de faible intensité. Revue de Physique Appliquée, 1968, 3 (3), pp.250-256. 10.1051/rphysap:0196800303025000 . jpa-00242856

HAL Id: jpa-00242856

https://hal.science/jpa-00242856

Submitted on 1 Jan 1968

HAL is a multi-disciplinary open access archive for the deposit and dissemination of scientific research documents, whether they are published or not. The documents may come from teaching and research institutions in France or abroad, or from public or private research centers.
L'archive ouverte pluridisciplinaire HAL, est destinée au dépôt et à la diffusion de documents scientifiques de niveau recherche, publiés ou non, émanant des établissements d'enseignement et de recherche français ou étrangers, des laboratoires publics ou privés. 


\title{
PHOSPHOROSGOPE SPEGTROPHOTOGRAPHIQUE DESTINÉ A LA MESURE DES DURÉES DE VIE DES LUMINESGENGES RETARDÉES DE FAIBLE INTENSITÉ
}

\author{
Par MM. Yvan ROUSSET, Françors DUPUY et Robert LOCHET, \\ Équipe associée au C.N.R.S., $n^{\circ}$ r6, Laboratoire d’Optique Moléculaire de la Faculté des Sciences de Bordeaux.
}

(Reçu le 24 juin 1968.)

Résumé. - L'emploi d'un spectrographe de grande luminosité s'impose dans l'étude des luminescences des cristaux moléculaires purs ou dopés, formées de différents spectres de faible intensité. Le disque unique d'un phosphoroscope à vitesse variable découpe à la fois une excitation d'intensité constante et monochromatisée, et la luminescence. Si $E$ désigne l'éclairement reçu par la plaque photographique, on montre qu'à la condition de choisir convenablement la vitesse $n$ de rotation du disque, la décroissance de $\log E / n$ en fonction de $1 / n$ est linéaire et permet de remonter au taux de déclin.

Comme application, on détermine la durée de vie de l'exciton triplet dans la benzophénone $\left(\tau \approx 1,8 \times 10^{-4} \mathrm{~s}\right)$ et l'anthracène $\left(\tau \approx 2,4 \times 10^{-4} \mathrm{~s}\right)$ cristallisés à $77^{\circ} \mathrm{K}$.

\begin{abstract}
The use of a spectrograph of great luminosity is necessary for studies of luminescence formed from different spectra of weak intensity emitted by pure or doped molecular crystals. A single disc of a phosphoroscope rotating at variable speed cuts the constant and monochromatic excitation beam and the emitted luminescence. $E$ being the light received by the photographic plate, we show, if the proper choice of disc rotation $n$ is made, that the decrease of $\log E / n$ as a function of $1 / n$ is linear and allows the calculation of the rate of the decrease of the luminescence.

As an application, we give the triplet exciton lifetime of crystallized benzophenone $\left(\tau \approx 1.8 \times 10^{-4} \mathrm{~s}\right)$ and anthracene $\left(\tau \approx 2.4 \times 10^{-4} \mathrm{~s}\right)$ at $77^{\mathrm{oK}}$.
\end{abstract}

Introduction. - L'excitation par éclairs brefs et la détection photoélectrique en liaison avec un oscillographe constituent un procédé très rapide de mesure du taux de déclin de la phosphorescence $\beta$ (émission $T \rightarrow S$ ) dans le cas des solutions rigidifiées à basse température. La durée de vie étant supérieure à $10^{-3} \mathrm{~s}$, on peut utiliser les éclairs de stroboscopes de type commercial dont la largeur à mi-hauteur ne dépasse pas $2 \mu s$. A l'aide de filtres optiques et de monochromateurs, on élimine dans le faisceau luminescent l'excitation et la fluorescence $\left(S^{*} \rightarrow S\right)$, et on n'est pas gêné par les queues de ces deux émissions qui peuvent ne pas être négligeables quelques millièmes de seconde après le maximum de l'excitation [1].

Une difficulté se présente, et le monochromateur devient inopérant si l'on veut étudier une émission retardée de même composition spectrale que la fluorescence directe, mais d'intensité beaucoup plus faible que celle-ci (phosphorescence $\alpha$ des colorants, fluorescence retardée due à l'annihilation de l'énergie électronique de deux triplets dans le cas des cristaux ou des solutions rigidifiées). La loi de décroissance avec le temps de la fluorescence directe suit pratiquement celle de l'éclair, et la queue de cette émission quasi instantanée qui n'est plus éliminée par le monochromateur peut être plus intense que certaines fluorescences retardées. Il en est couramment ainsi dans l'étude de la luminescence des cristaux moléculaires, purs ou dopés, qu'il s'agisse des fluorescences retardées ou des phosphorescences $T \rightarrow S$ des molécules du cristal ou des impuretés. Il faut donc supprimer la queue de l'éclair d'excitation et utiliser un obturateur mécanique pour découper le faisceau incident : ce sera le disque unique d'un phosphoroscope type Becquerel dont les deux séries de secteurs modulent respectivement le faisceau excitateur et le faisceau luminescent.

Avec la plupart des cristaux, surtout lorsqu'ils sont dopés, il existe plusieurs émissions retardées dont les spectres peuvent chevaucher; il faut donc en faire l'analyse spectrale et pour chacune des émissions isoler au moyen d'un monochromateur une bande étroite qui sera reçue par le photomultiplicateur; dans de nombreux cas, on est alors à la limite de la sensibilité de la détection photoélectrique.

Nous avons adopté en définitive la détection photographique et en faisant précéder notre spectrographe d'un phosphoroscope à vitesse réglable jusqu'à $6000 \mathrm{t} / \mathrm{mn}$, nous avons pu déterminer la durée de 
vie des émissions retardées de très nombreux cristaux purs ou dopés, lorsque cette durée de vie n'était pas inférieure à $10^{-4} \mathrm{~s}$.

Si la technique photographique est plus longue que la détection photoélectrique, elle a le double avantage :

1) D'être encore utilisable avec des émissions d'intensités trop faibles pour être mesurées avec un photomultiplicateur;

2) De permettre la mesure simultanée, dans les mêmes conditions d'excitation, des durées de vie des différentes émissions, et pour chacune d'elles d'opérer sur les différentes bandes spectrales qu'on leur attribue.

Principe de montage. - Pour la mesure de la durée de vie, nous verrons qu'il est fondamental que l'intensité de la source excitatrice ne varie pas et que l'on puisse opérer avec différentes vitesses de rotation du phosphoroscope. Il faut donc renoncer aux arcs au mercure haute pression qui, modulés à l'alternance du secteur, ne sont efficaces que si le disque du phosphoroscope est entraîné par un moteur synchrone. Nous avons adopté un arc court au xénon (XBO $450 \mathrm{~W}$ ). Un miroir ellipsoïdal en forme une image sur la fente $\mathrm{F}_{1}$ d'un monochromateur à prisme de quartz (fig. 1). Le rayonnement infrarouge est absorbé par l'eau distillée et dégazée d'une cuve hermétique de $5 \mathrm{~cm}$ d'épaisseur à faces de silice. Cette cuve et son contenu sont refroidis par une circulation d'eau froide. $\mathrm{Au}$ moyen d'un diaphragme de grand pouvoir déflecteur $\mathrm{D}$, on limite rigoureusement l'ouverture utile du faisceau admis dans le monochromateur à celle du miroir collimateur du monochromateur.

Le monochromateur est du type à déviation constante de $180^{\circ}$ (Trégor de Jobin et Yvon). Les objectifs sont des miroirs sphériques de distance focale $420 \mathrm{~mm}$. Le prisme en silice fondue a une hauteur de $9 \mathrm{~cm}$ et les faces atteignent $15 \mathrm{~cm}$ de longueur. En définitive, l'ouverture utile du monochromateur atteint $f / 3,2$.

C'est immédiatement contre la fente de sortie $\mathrm{F}_{2}$ du monochromateur ( fig. 2) que se trouve le plan du disque du phosphoroscope. La fente $\mathrm{F}_{2}$ se trouve au voisinage du centre de courbure du miroir sphérique $\mathrm{MS}_{\mathrm{E}}$; on obtient sur la poudre cristalline une bonne image de $\mathrm{F}_{2}$ en vraie grandeur après que, grâce à un miroir plan $\mathrm{MP}_{\mathrm{E}}$, le faisceau ait été dévié vers le bas.

Les rayons émis par cette poudre, excitation diffusée et luminescence recueillis par le miroir $\mathrm{MP}_{0}$, vont, après traversée de l'objectif $\mathrm{L}$, former une image de l'échantillon sur le disque. Après traversée du disque, les rayons lumineux sont repris par un miroir plan et un objectif pour former l'image définitive sur la fente du spectrographe. C'est par rotation de l'ensemble prisme-miroir plan du monochromateur que l'on règle la longueur d'onde excitatrice. Comme le faisceau excitateur ne rencontre que des miroirs, son réglage

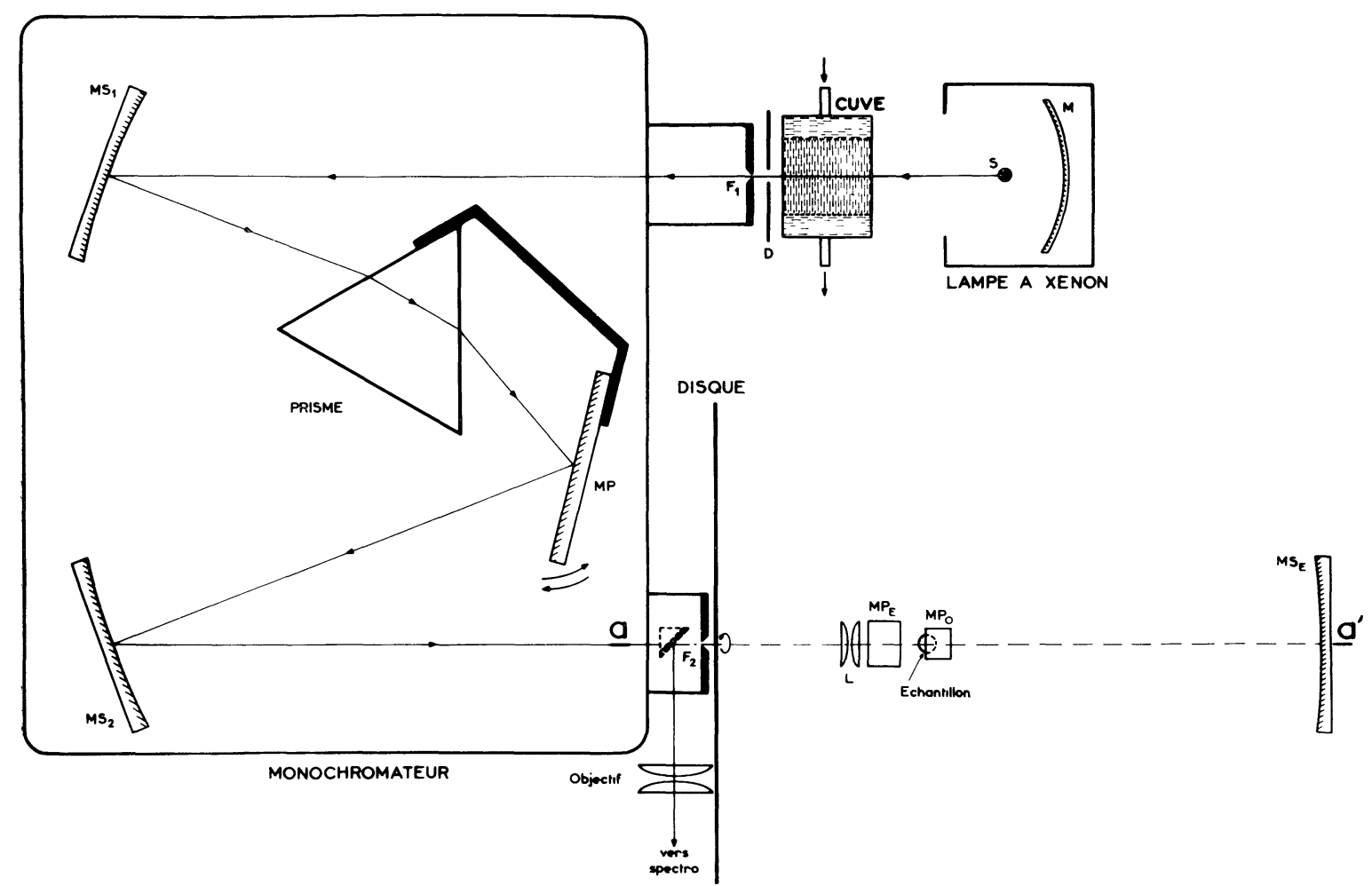

FIG. 1. 




FIG. 2.

n'a pas à être modifié quand on change de radiation excitatrice, depuis l'ultraviolet jusqu'au rouge.

Le cryostat est simplement constitué par un vase Dewar. L'azote liquide est surmonté d'une couche d'azote gazeux, sec, froid et dense qui déborde continuellement et évite la formation de givre sur l'échantillon et sur le trajet des faisceaux lumineux. En plongeant presque entièrement dans l'azote liquide le cylindre d'aluminium qui supporte la poudre cristalline, la température de l'échantillon se fixe au voisinage de $77^{\circ} \mathrm{K}$. En abaissant le dewar, une nouvelle température d'équilibre s'établit, d'autant plus élevée que la longueur émergente du cylindre est plus grande. Pour accentuer l'effet sur la température du déplacement du niveau de l'azote liquide, on peut donner au support d'échantillon une forme conique, la pointe dirigée vers le bas.

Le spectrographe est très lumineux : son objectif catadioptrique est ouvert à $f / 0,65$ avec une distance focale de $35 \mathrm{~mm}$. Son principe et sa description ont fait l'objet de publications antérieures [2] et [3]. Rappelons ici que, malgré les dimensions réduites du châssis photographique, on peut obtenir 8 spectres sur une même pellicule circulaire de $18 \mathrm{~mm}$ de diamètre; cette performance est importante pour la suite des mesures photométriques.

Les durées de fermeture de la fente du monochromateur (faisceau excitateur) et de l'image de la fente du spectrographe (faisceau luminescent) qui s'ajoutent au temps mort entre la fin de l'excitation et le début de l'observation doivent être aussi courtes que possible, lorsqu'on étudie des luminescences dont la durée de vie est voisine de $10^{-4} \mathrm{~s}$; on doit donc opérer avec de grandes vitesses de l'obturateur; on utilise des disques de grands diamètres $(320 \mathrm{~mm})$ animés de grandes vitesses de rotation, jusqu'à $6000 \mathrm{t} / \mathrm{mn}$. Ces disques parfaitement équilibrés découpés dans une feuille d'aluminium très mince $(1 \mathrm{~mm})$ sont soigneusement calés et centrés sur l'arbre d'un puissant moteur $(1 / 6 \mathrm{ch})$ à courant continu dont la vitesse est stabilisée pour toute valeur choisie à l'avance comprise entre 6 et $6000 \mathrm{t} / \mathrm{mn}$. Nous avons utilisé le varistor de la Compagnie Hugonnot.

L'asservissement est obtenu par une alimentation du moteur en courant redressé : une tension de consigne correspondant à la vitesse de rotation désirée et affichée à l'aide d'un potentiomètre est opposée à la tension délivrée par une dynamo tachymétrique 
calée sur l'arbre du moteur. L'écart entre ces deux tensions est considérablement amplifié. Le signal obtenu, après une mise en forme convenable, positionne la phase d'une impulsion de manière à modifier dans le sens voulu la durée, d'une fraction de seconde, pendant laquelle le redresseur (Thyristor) est conducteur. La stabilité de la vitesse de rotation est voisine de $0,25 \%$.

Principe de la mesure des durées de vie. - Le disque est percé de deux séries de secteurs que traversent l'une le faisceau excitateur, l'autre le faisceau luminescent. L'équilibrage du disque impose un nombre pair de secteurs.

La figure 3 représente un disque percé de 4 secteurs

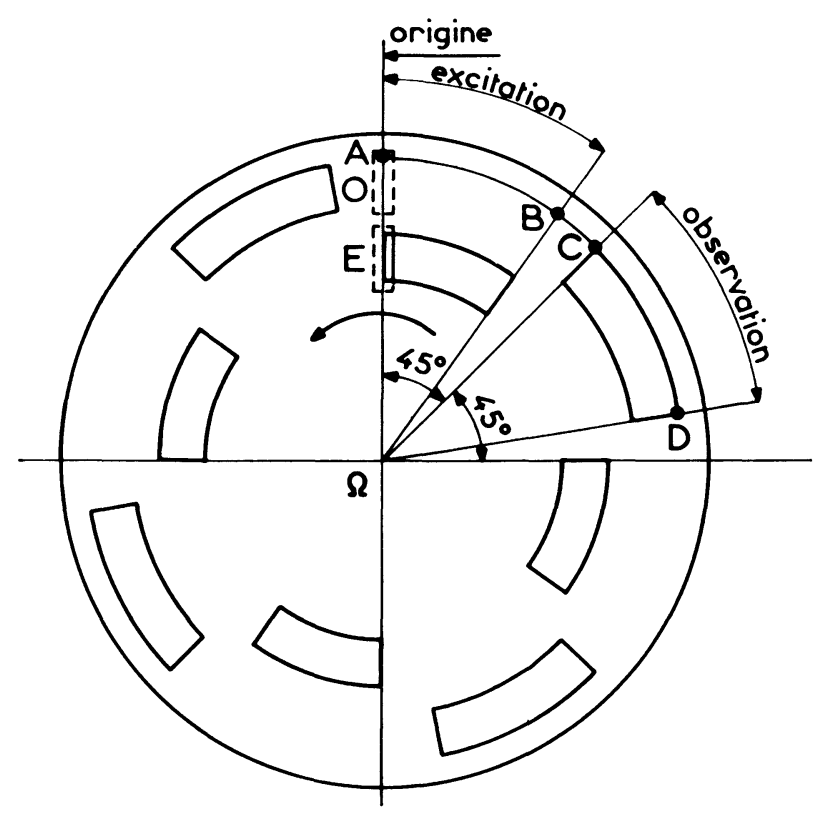

FIG. 3.

d'excitation et 4 secteurs d'observation d'angles au centre sensiblement égaux et voisins de $\pi / 4$.

En prenant pour origine des temps le début de l'excitation, nous désignerons par $t_{\mathrm{e}}$ la durée de l'excitation, par $t_{1}$ le début de l'observation et par $t_{2}$ la fin de l'observation. Ces durées sont reliées aux angles au centre correspondants pris en radians par :

$$
t_{\mathrm{e}}=\frac{\mathrm{A} \hat{\Omega} \mathrm{B}}{2 \pi N}, \quad t_{1}=\frac{\mathrm{A} \hat{\Omega} \mathrm{C}}{2 \pi N}, \quad t_{2}=\frac{\mathrm{A} \hat{\Omega} \mathrm{D}}{2 \pi N}
$$

où $N$ est le nombre de tours par seconde.

Supposons d'abord infiniment étroits les faisceaux excitateur et luminescent. Nous caractérisons le faisceau excitateur par son intensité constante $I_{0}$. Décomposons chaque éclair de durée $t_{\mathrm{e}}$ en éclair élémentaire de durée $\mathrm{d} \theta$ caractérisé par leur date $\theta$

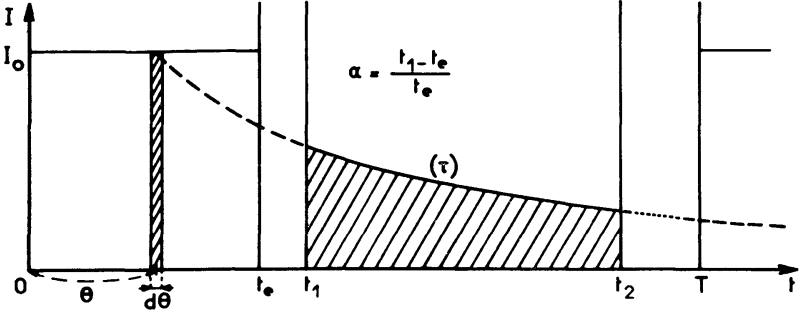

FIG. 4.

comptée à partir du début de l'excitation (fig. 4). Chacun de ces éclairs d'énergie $I_{0} \mathrm{~d} \theta$ porte dans l'état excité le même nombre de molécules, si l'on admet que le nombre des molécules dans l'état triplet n'est qu'une faible fraction du nombre de molécules dans l'état fondamental. On peut donc poser qu'en se désactivant avec une durée de vie $\tau$, ces molécules envoient entre les instants $t_{1}$ et $t_{2}$ un flux $\mathrm{d} \theta$ proportionnel à :

$$
\frac{I_{0}}{\tau} \mathrm{d} \theta \int_{t_{1}-\theta}^{t_{2}-\theta} \mathrm{e}^{-t / \tau} \mathrm{d} t=I_{0} \cdot \mathrm{d} \theta \cdot \mathrm{e}^{\theta / \tau}\left(\mathrm{e}^{-t_{1} / \tau}-\mathrm{e}^{-t_{2} / \tau}\right)
$$

et pour l'ensemble des éclairs élémentaires émis entre l'origine des temps et la fin de l'excitation $t_{\mathrm{e}}$ :

$$
\begin{aligned}
\Phi_{0}= & I_{0}\left(\mathrm{e}^{-t_{1} / \tau}-\mathrm{e}^{-t_{2} / \tau}\right) \int_{0}^{t_{\theta}} \mathrm{e}^{\theta / \tau} \cdot \mathrm{d} \theta \\
& =\tau I_{0}\left(\mathrm{e}^{t_{\theta} / \tau}-1\right)\left(\mathrm{e}^{-t_{1} / \tau}-\mathrm{e}^{-t_{2} / \tau}\right) \\
& =\tau I_{0} \mathrm{e}^{-\left(t_{1}-t_{\theta}\right) / \tau}\left[1-\mathrm{e}^{-t_{\theta} / \tau}\right]\left[1-\mathrm{e}^{-\left(t_{2}-t_{1}\right) / \tau}\right] .
\end{aligned}
$$

Posons $t_{1}=t_{\mathrm{e}}(1+\alpha)$, le produit $\alpha t_{\mathrm{e}}$ représente le temps mort entre la fin de l'excitation et le début de l'observation. Si, comme il est courant, les angles d'ouverture de l'excitation et de l'observation sont pris égaux, c'est-à-dire si $t_{2}-t_{1}=t_{\mathrm{e}}$, il vient :

$$
\Phi_{0}=\tau I_{0} \mathrm{e}^{-\alpha t_{\theta} / \tau}\left(1-\mathrm{e}^{-t_{\mathrm{e}} / \tau}\right)^{2} \text {. }
$$

On peut alors écrire que l'excitation restant constante, le flux moyen par seconde (ou encore l'énergie $E$ reçue par la plaque photographique par unité de temps) est donné par :

$$
E=I_{0} \mathrm{e}^{-\alpha t_{\mathrm{e}} / \tau} \frac{\left(1-\mathrm{e}^{-t_{\mathrm{e}} / \tau}\right)^{2}}{t_{\mathrm{e}} / \tau} .
$$

L'éclairement reçu par la plaque photographique est donc lié très simplement à $t_{\mathrm{e}}$ et à $\alpha$, et donc pour un disque déterminé à la vitesse de rotation du disque.

Nous allons maintenant justifier la valeur 0,2 que nous avons adoptée pour $\alpha$. Dans l'hypothèse des sections infiniment petites pour les faisceaux excitateur et luminescent, la détermination de $\alpha$ se réduirait à la mesure d'un angle au centre, que l'on pourrait prendre aussi petit que l'on voudrait de façon à augmenter l'éclairement $E$ dans le cas des luminescences de très courte durée de vie. Mais la largeur finie de l'ordre du millimètre des faisceaux excitateur et luminescent se traduit par une erreur systématique dans l'évaluation de $\alpha$; cette erreur étant indépendante de la vitesse de rotation, on ne peut la diminuer 
en valeur relative qu'en augmentant $\alpha$, c'est-à-dire le retard $t_{1}-t_{\mathrm{e}}$; corrélativement, on ne reçoit sur le spectrographe qu'une fraction de plus en plus faible du flux luminescent. Si l'on veut limiter à $10 \%$ l'erreur systématique sur $\alpha$, compte tenu des largeurs des faisceaux, il faut que la longueur de l'arc BC soit au moins égale à $13 \mathrm{~mm}$, ce qui, avec un secteur voisin de $\pi / 4$, entraîne la valeur 0,20 pour $\alpha$.

Nous avons tracé une série de courbes représentatives de $\log \left(E t_{\mathrm{e}} / \tau\right)=f\left(t_{\mathrm{e}} / \tau\right)$ pour des valeurs différentes de $\alpha$. Au-delà du maximum, la décroissance est quasi rectiligne avec la pente $\alpha$ (fig. 5). Si l'on se fixe $t_{\mathrm{e}} / \tau$, c'est-à-dire pour $\tau$ déterminé, une vitesse de

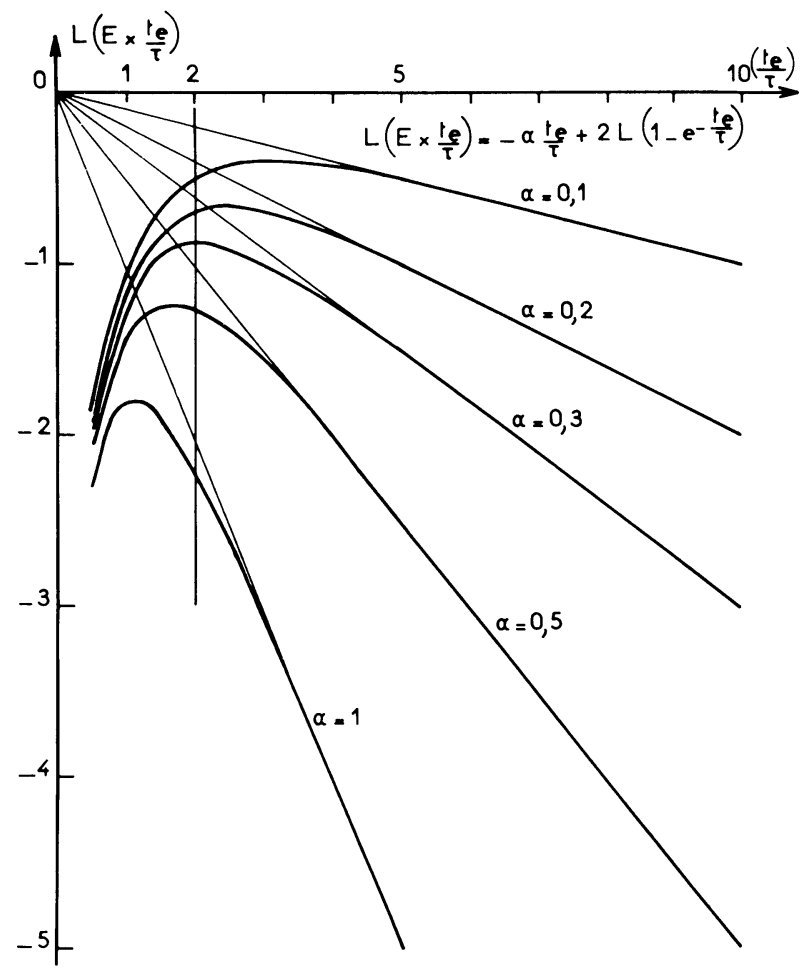

FIG. 5.

rotation constante (par exemple : $t_{\mathrm{e}} / \tau=2$ ), l'ordonnée, donc le flux lumineux reçu par le spectrographe, est d'autant plus faible que $\alpha$ est plus grand. On vérifie que l'on a intérêt à diminuer le plus possible $\alpha$, mais nous avons vu que l'on ne pouvait pas descendre au-dessous de 0,20 , si l'on fixe à moins de $10 \%$ la limite de l'erreur relative sur ce paramètre.

Si maintenant on trace la courbe $\log \left(E t_{\mathrm{e}}\right)$ en fonction de $t_{\mathrm{e}}$, on retrouve une décroissance linéaire, mais en valeur absolue la pente est égale à $\alpha / \tau$.

Avec le disque que nous avons utilisé, $\alpha=0,20$, le temps d'excitation en secondes se déduit de la vitesse de rotation $n \mathrm{t} / \mathrm{mn}$, par la relation :

$$
t_{\mathrm{e}(\mathrm{s})}=6,3 / n \text {. }
$$

Finalement, on tracera le graphique $\log _{10} E / n$ en fonction de $1 / n$ et si $p$ est la valeur absolue de la pente de la partie rectiligne, il vient en définitive :

$$
\tau_{(\mathrm{s})}=0,55 / p \text {. }
$$

Il n'y a aucune ambiguïté si les points expérimentaux $\log _{10} E / n, 1 / n$ se placent sur une droite de pente négative. Mais ce n'est pas le cas pour certains d'entre eux au voisinage du maximum de la courbe théorique de la figure 5, soit parce que la vitesse de rotation du disque est trop rapide, soit parce que la durée de vie est relativement longue; c'est-à-dire si $t_{\mathrm{e}} / \tau$ est inférieur à 3 . On peut en déduire immédiatement une valeur approchée de la durée de vie et adopter pour la vitesse du disque des valeurs correspondant à la partie rectiligne de la courbe.

Soit $n_{0}$ l'abscisse du maximum. L'étude de la fonction $\log \left(E t_{\mathrm{e}} / \tau\right)=\log \left[\mathrm{e}^{-\alpha t_{\mathrm{e}} / \tau}\left(1-\mathrm{e}^{-t_{\mathrm{e}} / \tau}\right)^{2}\right]$ montre que le maximum s'obtient pour $t_{\mathrm{e}} / \tau=\log 2(1+\alpha) / \alpha$, soit pour $t_{\mathrm{e}} / \tau=2,5$ si $\alpha=0,20$, ou enfin en introduisant la vitesse de rotation $n$ en $\mathrm{t} / \mathrm{mn}$ :

$$
\tau_{\mathrm{s}} \simeq 6,3 / 2,5 n_{0} \simeq 2,5 / n_{0} .
$$

Remarque. - Si l'on veut tenir compte de la périodicité $T$ du phénomène en admettant que l'extinction de la luminescence n'est pas complète avant une nouvelle excitation, ou plutôt que l'on observe pendant le temps $t_{2}-t_{1}$, non seulement la luminescence produite par l'éclair précédent, mais également celle qui était produite par les éclairs aux dates $T, 2 T, 3 T$ avant le dernier, on doit alors écrire au lieu de la relation (1) :

$$
\begin{aligned}
& \Phi=\tau I_{0}\left(\mathrm{e}^{t_{\mathrm{e}} / \tau}-1\right) \sum_{i}\left(\mathrm{e}^{-\left(t_{1}+i T\right) / \tau}-\mathrm{e}^{-\left(t_{2}+i T\right) / \tau}\right) \\
& \qquad=0,1,2, \ldots \infty \\
& \Phi=\Phi_{0} \sum_{i} \mathrm{e}^{-i T / \tau}=\Phi_{0} \frac{1}{1-\mathrm{e}^{-T / \tau}} \\
& \Phi \simeq \Phi\left(1+\mathrm{e}^{-T / \tau}\right) .
\end{aligned}
$$

Comme la période $T$ est légèrement supérieure à $2 t_{\mathrm{e}}$ (en effet, $T=t_{\mathrm{e}}+\alpha t_{\mathrm{e}}+t_{2}-t_{1}$ ), le facteur correctif $\mathrm{e}^{-T / \tau}$ n'atteint $10 \%$ que si $t_{\mathrm{e}} / \tau<1$; or nous avons vu que l'on doit toujours opérer avec des vitesses telles que le rapport $t_{\mathrm{e}} / \tau$ soit supérieur à 2,5 . On peut donc dans tous les cas négliger l'effet des éclairs antérieurs.

Application : luminescence de la benzophénone cristallisée à $19 \%$ o K. - Nous disposons de benzophénone qui ne présente plus, à l'état cristallisé, la phosphorescence verte (spécifique d'une impureté, tant par sa durée de vie que par la fréquence de la bande $(0,0)$ et sa structure vibrationnelle).

La luminescence du cristal est bleue, nous en avons étudié la composition spectrale et le taux de déclin à la température de $77 \mathrm{oK}$. Les échantillons étudiés à l'état de poudre étaient prélevés en différents points 
de la colonne soumise à la fusion de zones après environ 60 passages.

Avec le montage que l'on vient de décrire, pour une vitesse de rotation du disque de $2000 \mathrm{t} / \mathrm{mn}$ et la fente du spectrographe étant ouverte à $100 \mu$, on obtient un bon spectre en moins de $3 \mathrm{mn}$ de pose. Suivant l'échantillon utilisé, on obtient le spectre I ou le spectre II ( fig. 6). Le spectre I, analogue à celui obtenu

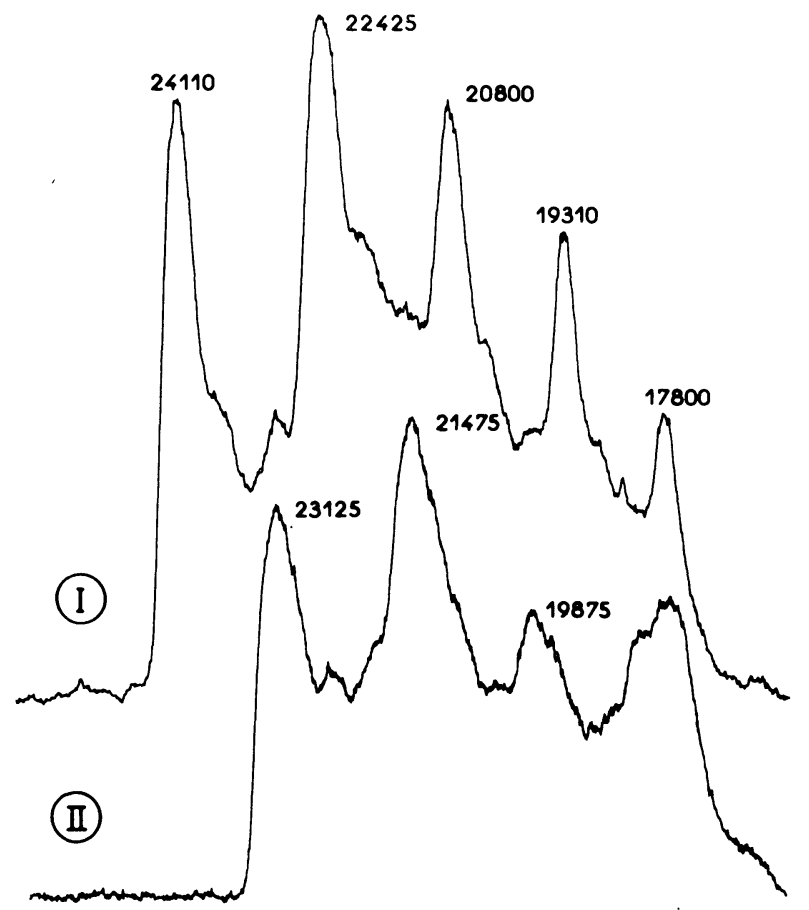

FIG. 6.

à $20 \mathrm{oK}$ sans phosphoroscope par D. S. McClure et P. L. Hanst [4], est identique à celui de l'émission de la benzophénone en solution solide dans l'E.P.A. ou dans le cyclohexane à $77^{\circ} \mathrm{K}$. Les bandes du spectre II peuvent se déduire de celles du spectre I par un déplacement de $1000 \mathrm{~cm}^{-1}$ vers les grandes longueurs d'onde.

Mesures. - Avec les échantillons donnant le spectre I, si l'on fait l'étude de l'intensité des bandes en fonction de la vitesse de rotation du disque, on obtient les courbes de la figure 7 . Ces courbes relatives à chacune des deux bandes 22425 et $24110 \mathrm{~cm}^{-1}$ suggèrent pour cette émission deux processus de durées de vie différentes :

$$
\tau_{\mathrm{I}}=1,8 \times 10^{-4} \mathrm{~s} \quad \text { et } \quad \tau_{\mathrm{I}}^{\prime}=1,2 \times 10^{-3} \mathrm{~s} .
$$

Les ordonnées à l'origine, extrapolées pour $n$ infini, sont proportionnelles à $\tau_{I} I_{0}$ et $\tau_{I}^{\prime} I_{0}^{\prime}$ (voir relation $(2)$ ), où $I_{0}$ et $I_{0}^{\prime}$ sont les flux que l'on recevrait par seconde en l'absence de phosphoroscope. Comme ici :

$$
\log \frac{E_{0}}{n}-\log \frac{E_{0}^{\prime}}{n} \simeq 1
$$



FIG. 7.

on en déduit $I_{0}^{\prime}=\frac{2}{300} I_{0}$, c'est-à-dire que l'intensité de la composante lente représente moins de $1 \%$ de l'intensité totale de l'émission.

En opérant sur un échantillon qui donne le spectre II, on augmente la vitesse sur les clichés obtenus aux grandes vitesses, le spectre I vient s'ajouter au spectre II. On peut alors, grâce au spectrographe, faire simultanément l'étude de la bande $24110 \mathrm{~cm}^{-1}$ du spectre I et celle des bandes 21475 et $23125 \mathrm{~cm}^{-1}$ du spectre II (fig. 8). De la partie rectiligne des courbes relatives aux bandes du spectre II, on déduit

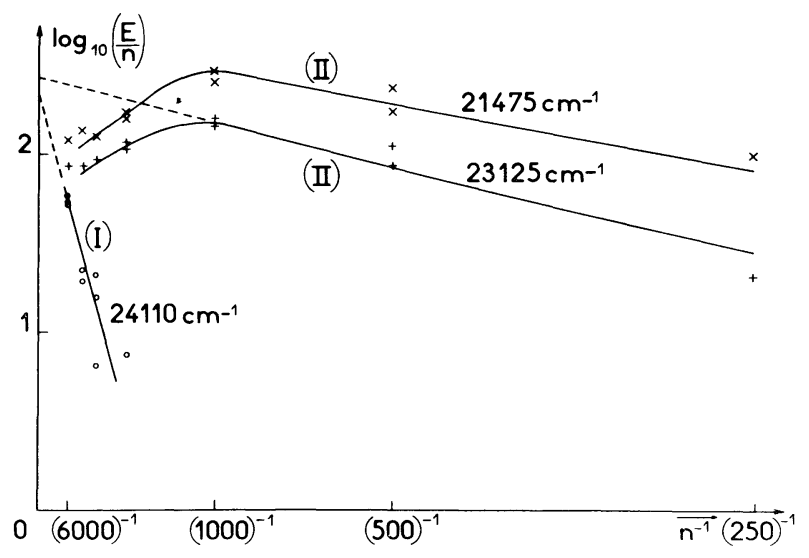

FIG. 8.

une durée de vie moyenne $\tau_{\mathrm{II}}=2,6 \times 10^{-3} \mathrm{~s}$. Remarquons que l'abscisse $\frac{1}{n_{0}}$, avec $n_{0}=1000 \mathrm{t} / \mathrm{mn} \mathrm{du}$ maximum de la courbe conduit à la même valeur pour la durée de vie. Sur le même cliché donc, on a retrouvé pour la bande $24110 \mathrm{~cm}^{-1}$ la durée de vie $\left(\tau_{\mathrm{I}}=1,5 \times 10^{-4} \mathrm{~s}\right)$ de la composante rapide $\mathrm{du}$ spectre I. A partir des ordonnées à l'origine des droites, on déduit de la même façon que précédemment qu'en l'absence de phosphoroscope le spectre I 
est 20 fois plus intense que le spectre II. Ce résultat explique que, dans ces conditions, on comprend qu'il soit très difficile d'observer nettement les bandes du spectre II à côté de celles du spectre I. L'intensité du spectre II est très variable d'un échantillon à l'autre; et en particulier, si l'on utilise certaines parties monocristallines de l'extrémité de la colonne, le spectre II a disparu. Mais si ces échantillons sont fondus et brusquement refroidis dans l'azote liquide, le spectre II apparaît avec une forte intensité. Nous en avons déduit que cette émission était liée à la formation, dans le réseau cristallin, de défauts dont le nombre par unité de volume dépend des circonstances de la solidification [5]. C'est donc de la décroissance du spectre I que l'on peut déduire la durée de vie $\tau=1,8 \times 10^{-4} \mathrm{~s}$ pour l'exciton triplet de la benzophénone cristallisée à $77 \mathrm{oK}$.

Cas de l'anthracène cristallisé. - Nous avons opéré sur un anthracène cristallisé pur, qui ne présente plus, $\mathrm{au}$ phosphoroscope, à température ordinaire l'émission verte $\mathrm{S}^{*} \rightarrow \mathrm{S}$ du naphtacène impureté.

Quelles que soient les températures $\left(300{ }^{\circ} \mathrm{K}\right.$ ou $77 \mathrm{oK}$ ) et les excitations utilisées (U.V. ou jaune), nous avons toujours opéré sur les bandes bleues caractéristiques d'une transition $S^{*} \rightarrow S$ de l'anthracène, identiques à celles de la fluorescence directe du cristal d'anthracène à la même température.

Mesures. - A $300 \mathrm{oK}$, avec une bande d'excitation centrée sur $380 \mathrm{~m} \mu$ (fig. 9), nous trouvons que la luminescence a une durée de vie de $0,75 \mathrm{~ms}$. Ce résultat est en parfait accord avec celui que nous avions obtenu pour l'émission du naphtacène dissous dans l'anthracène, dans l'hypothèse où l'énergie électronique reste piégée avant de remonter dans la bande de l'exciton singulet [6].

A 77 oK, avec la même excitation, de l'étude de la décroissance de l'émission on déduit deux durées de vie $\tau=1,2 \times 10^{-4} \mathrm{~s}$ et $\tau^{\prime}=5,5 \times 10^{-4} \mathrm{~s}$. L'extrapolation des droites $\log (E / n)=f(1 / n)$ montre que l'intensité de l'émission de longue durée de vie représente seulement $3 \%$ de l'intensité totale. Si l'on pose que cette émission $\mathrm{S}^{*} \rightarrow \mathrm{S}$ ne provient que de l'annihilation des excitons triplets [7], on en déduit pour cet exciton une durée de vie $\tau_{\mathrm{T}} \approx 2,4 \times 10^{-4} \mathrm{~s}$.

On retrouve d'ailleurs cette même durée de vie avec l'anthracène cristallisé à $77 \mathrm{oK}$ à partir d'une bande excitatrice centrée sur $5800 \AA$ (qui coïncide avec un maximum d'absorption «interdite» $T \leftarrow S[8]$ ). A cette émission de courte durée de vie succède une

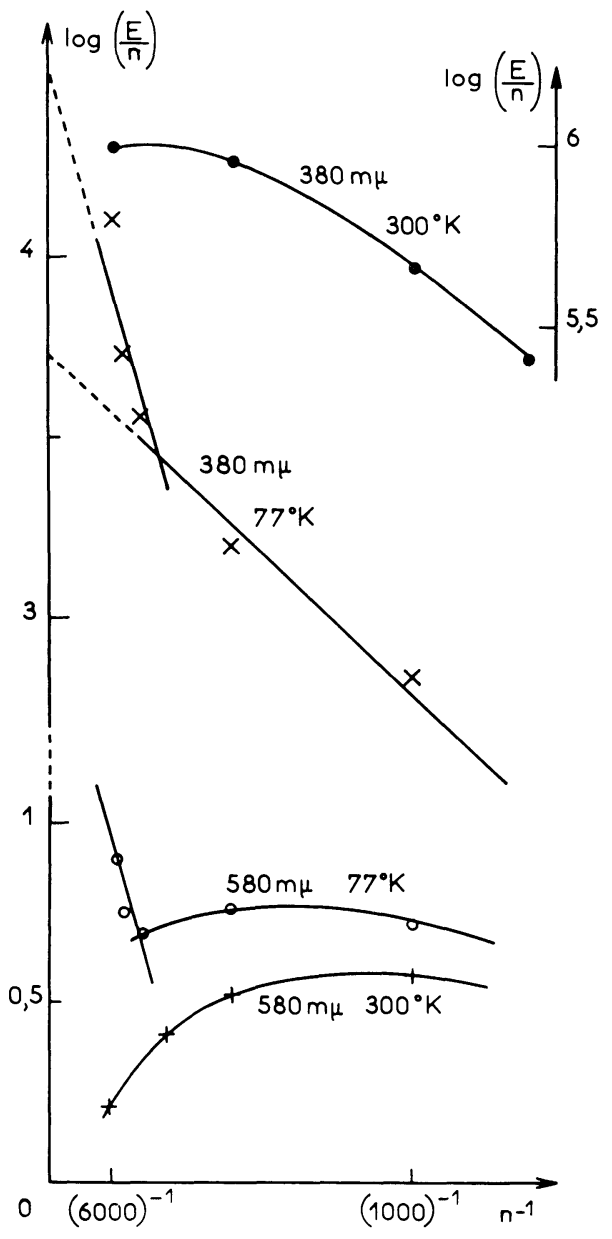

FIG. 9

émission dont la durée de vie atteint la milliseconde.

A $300{ }^{\circ} \mathrm{K}$, et toujours l'excitation jaune, on n'observe plus à travers le phosphoroscope que la seconde émission, car à la température ordinaire la durée de vie de l'exciton triplet est devenue trop courte.

Si l'on fixe à $6000 \mathrm{t} / \mathrm{mn}$ la vitesse de rotation du phosphoroscope, la luminescence excitée par l'U.V. est environ 80 fois plus intense à température ordinaire qu'à basse température. Par contre, avec l'excitation jaune, si l'on se limite à l'étude des émissions de longue durée de vie, les intensités ne varient pas avec la température, mais elles sont environ 1000 fois plus faibles que celles obtenues à $77 \mathrm{oK}$ avec l'excitation U.V.

\section{BIBLIOGRAPHIE}

[1] Martinez (A.), J. Physique, 1963, 24, 117 A.

[2] LOCHETi(R.) etCLASTRE (J.), Rev. Optique, 1950, 29, 273.

[3] LoCHET (R.) et Rousseir (Y.), J . Physique, 1963, 24, 286.

[4] MCClure (D. S.) et Hanst (P. L.), J. Chem. Physics, $1955,23,1772$
[5] Rousseit (Y.), C. R. Acad. Sc., 1968, 266, 1600.

[6] Rousseit (Y.), C. R. Acad. Sc., 1964, 258, 4687.

[7] Rousseit (Y.), C. R. Acad. Sc., 1965, 260, 6834.

[8] Avakian (P.), Abramson (E.), KePleR (R. G.) et CARIS (J. C.), J. Chem. Physics, 1963, 39, 1127. 